\title{
COMPARATIVE STUDY OF COLOR DOPPLER VOIDING UROSONOGRAPHY WITHOUT CONTRAST ENHANCEMENT AND DIRECT RADIONUCLIDE VOIDING CYSTOGRAPHY FOR THE DIAGNOSIS OF VESICOURETERIC REFLUX IN CHILDREN
}

\author{
M. Mohammadi fallah ${ }^{1}$, M. Falahati ${ }^{1}$, A. Mohammadi ${ }^{1}$, A.A. Nikibakhsh ${ }^{2}$, M. Ghasemi-Rad ${ }^{3}$ \\ ${ }^{1}$ Urmia University of Medical Sciences, ${ }^{2}$ Pediatric Nephrology, ${ }^{3}$ Student Research Committee, Urmia \\ University of Medical Sciences, Urmia, Iran
}

Objectives: We aimed to compare color DUS without the application of contrast enhancement and direct radionuclide voiding cystography (DRC) to assess the capability of color DUS without contrast in the detection and grading of VUR with respect to the sex and age of patients, as well as the severity of VUR.

Material and methods: In the 66 patients enrolled (56 girls, 10 boys), 132 reno-ureteral units were investigated. All patients underwent DUS and DRC within 3 hours. DRC was used as the reference standard.

Results: Our results indicate a good overall sensitivity (83\%) and specificity (77\%) of color DUS without contrast enhancement in comparison with DRC. As the age of the patients decreased, the sensitivity of DUS increased, reaching $100 \%$ among patients below one year of age. Upon the comparison of different grades of VUR severity, we found that sensitivity and specificity elevated as the grade increased (both parameters reaching $100 \%$ in the most severe cases).

Conclusion: We have demonstrated that DUS is a comparably reliable method to DRVC in the detection and grading of VUR especially at higher grades of VUR, even without the application of echo contrast agents. In high grades of VUR, the diagnosis and follow-up can be safely and reliably performed without the application of an US contrast medium. However, for an accurate diagnosis and grading, especially at lower grades, the application of a contrast medium can be recommended when US is performed. 